\title{
Prescribing Pattern for Skin Diseases in Dermatology OPD at Borumeda Hospital, North East, Ethiopia
}

\author{
Abebaw Tegegne ${ }^{1 *}$, Fentaw Bialfew ${ }^{2}$ \\ ${ }^{1}$ Pharmacologist in Borumeda Hospital, Dessie, Ethiopia \\ ${ }^{2}$ Health Officer in Borumeda Hospital, Dessie, Ethiopia \\ Email: mabebaw12@gmail.com, fentawbi@gmail.com
}

How to cite this paper: Tegegne, A. and Bialfew, F. (2018) Prescribing Pattern for Skin Diseases in Dermatology OPD at Borumeda Hospital, North East, Ethiopia. Pain Studies and Treatment, 6, 1-8. https://doi.org/10.4236/pst.2018.61001

Received: November 18, 2017

Accepted: December 30, 2017

Published: January 2, 2017

Copyright $\odot 2018$ by authors and Scientific Research Publishing Inc. This work is licensed under the Creative Commons Attribution International License (CC BY 4.0).

http://creativecommons.org/licenses/by/4.0/

\begin{abstract}
Introduction: Skin diseases are the major contributors of disease burden in society. Dermatological therapy ultimate goal is achieved by administering the safest and least number of drugs. The problem gets compounded with the inappropriate and irrational use of medicines. Therefore, periodic prescription audit in the form of prescribing patterns is away to improve irrational prescription. The objective of this study to assess the prescription patterns of dermatological agents in Borumeda hospital. Method: Hospital based retrospective cross sectional study in which prescribing patterns of dermatological agents are assessed. A total of 385 samples of patient record prescription from November/1/2016 to December/30/2016, and the sample were selected by systematic random sampling technique. Sample prescriptions were reviewed using structural data collection format. The Collected data was analyzed by using SPSS version 20. Result: Regarding rout of administration, the maximum number of drugs was prescribed topically (66.2\%). Topical steroids were the most commonly prescribed drugs (25.3\%). Use of generic prescribing in single drug prescribing was $81.7 \%$. The prevalence of atopic dermatitis was higher $(26.3 \%, 20.8 \%)$ in both male and female respectively followed by scabies in male with $12.2 \%$ and Acne vulgaris (12.9\%) in female. Number of drugs per prescription was higher (2.46) than WHO standard $(<2)$. Conclusion: The current study reveals that topical corticosteroids were commonly prescribed drugs in the dermatology unit and the prescribing practice imitates incidence of polypharmacy.
\end{abstract}

\section{Keywords}

Borumeda Hospital, Dermatological Agents, Prescription Patterns 


\section{Introduction}

Skin disease refers to disorders exclusively of the superficial layers of the skin. The commonest skin disorders seen in developing countries are psoriasis and scabies, but they have not been regarded as a significant problem that could benefit from public health measures. Most skin diseases are chronic and require lifetime treatment [1].

Improved quality of life by enhancing standards of medical treatment at all levels of the health care delivery system. Setting standards and assessing the quality of care through performance review should become part of everyday clinical practice [2].

Prescribing patterns studies are useful to monitor evaluate and suggest modifications in prescribers' prescribing habits so as to make medical care rational and cost effective. Such analysis not only improves the standards of medical treatment at all levels in the health system, but also supports in the identification of drug use related problems such as, drug-drug interaction, poly-pharmacy and adverse drug reaction [3] [4].

Irrational prescribing has a serious impact on health and economy, resulting in wastage of resource. The patterns of drug use in a hospital setting need to be monitored intermittently in order to analyze their rationality and increase the therapeutic benefits and reduce adverse effect [5].

\section{Methods}

\subsection{Study Area and Period}

Borumeda Hospital (BMH) is found in Eastern Amhara South Wollo zone, Dessie town, which is found about $411 \mathrm{~km}$ away from Addis Ababa, the capital city of Ethiopia. It was established in 1947 E.C through the support of missionary organization that was primary focused on dermatology and ophthalmic services. It has a total of 80 beds among these 30 beds is in dermatological ward. The study was conducted from November 01/2016-December 30/2016.

\subsection{Study Design and Study Population}

A retrospective institutional based quantitative cross sectional study was conducted by reviewing prescriptions and the sample were selected by systematic random sampling technique. The study population of this study was all dermatological patients who were visit $\mathrm{BMH}$, dermatology unit in outpatient department and for whom medicines were prescribed during study period.

\subsection{Sampling Technique and Sample Size}

\subsubsection{Sample Size}

The sample size for this particular study was calculated using formula for a single population proportion considering the following assumptions: A $95 \%$ confidence level, margin of error (0.05) and assume maximum variability $(p=0.5)$. These parameters are substituted in the following single population proportion 
formula;

$$
n=\frac{z^{2} p(1-p)^{2}}{d^{2}} \frac{(1.96)^{2} \times 0.5(1-0.5)^{2}}{(0.05)^{2}}=384
$$

where, $n=$ Sample size

$p=$ Proportion of people

$d=$ margin of error $(0.05)$

$z=$ confidence level $95 \%$ (i.e. 1.96)

The population of annual dermatological patients at BMH is $<10,000$, therefore sample size adjustment is needed by using population correction formula

$$
n_{o}=n i /[1+(n i / N)]
$$

where $N=8400$ (total number dermatological patients annually)

$n i=$ unadjusted sample size calculated Sample size

$n_{o}=$ adjusted Sample size $n_{o}$ is equal to $367 \%$ and $5 \%$ for (incomplete information or data) makes the sample size 385 .

\subsubsection{Sampling Technique}

After sample size determined, Systematic random sampling technique was used to select the sample prescription.

\subsection{Data Collection and Data Analysis}

Well-trained pharmacy personnel collected data on patient prescriptions. The specific types of data necessary to measure the prescription patterns were recorded for each patient prescription and entered directly into an ordinary prescription patterns form. After collection of the data, the data was entered in to SPSS version 20 for analysis.

\subsection{Quality Control}

The data was verified by well-trained pharmacy personnel through direct supervision of principal investigators during the data Collection period.

\subsection{Ethical Consideration}

The study was approved by chief executive officer, and the head of the pharmacy department in the BMH. A formal letter was written from $\mathrm{BMH}$ and given to pharmacy department and also confidentiality of the information obtained from the patient's prescription reviewed throughout the study period was kept.

\section{Result}

The study includes a total of 385 patients and overall numbers of male patients were 192 (49.9\%) while numbers of female patients were 193 (50.1\%). The maximum numbers of patients were in male between the age group of $21-30$ years $(25.5 \%)$ and in female $21-30$ years $(32.6 \%)$. The minimum numbers of patients were in male between Female $>60$ years $(1.6 \%)$ (Table 1 ). 
Table 1. Age with Sex distribution of the patients in dermatology unit of BMH, Dessie, 2016.

\begin{tabular}{ccc}
\hline Age group & Male, $\mathrm{n}(\%)$ & Female, $\mathrm{n}(\%)$ \\
\hline $0-10$ & $31(16.2 \%)$ & $30(15.5 \%)$ \\
$11-20$ & $47(24.5 \%)$ & $53(27.5 \%)$ \\
$21-30$ & $49(25.5 \%)$ & $63(32.6 \%)$ \\
$31-40$ & $19(9.9)$ & $22(11.4)$ \\
$41-50$ & $20(10.4 \%)$ & $15(7.8 \%)$ \\
$51-60$ & $14(7.3 \%)$ & $7(3.6 \%)$ \\
$>60$ & $12(6.3 \%)$ & $3(1.6 \%)$ \\
Total & 192 & 193 \\
\hline
\end{tabular}

Most of topical steroids were prescribed by generic name. The number of drugs prescribed by brand name was $18.3 \%$ for drugs prescribed in single and $15.6 \%$ for drug prescribed in fixed dose combinations (Table 2).

Out of 1104 drugs, topical route $731(66.2 \%)$ was the most route of administration. oral route $212(19.2 \%)$ was the second most route of administration followed by a combination systemic and local route of administration (Table 3).

Total number of drugs in 385 patients was found to be 946 . Number of drugs per prescription varied from 1 to 7 with average of 3.0. Most of the prescriptions consists of minimum of 2 drugs (180 prescriptions, 46.75\%). And the others with $14.80 \%, 22.60 \%$ contain a single drug and 3 drugs respectively (Table 4 ).

Based on Disease distribution, out of 385 patients, prevalence of atopic dermatitis was higher in with $(26.3 \%, 20.8 \%)$ both male and female respectively followed by scabies in male with $12.2 \%$ and Acne vulgaris (12.9\%) in female (Table 5).

The drugs prescribed, topical steroids were the most commonly used (25.3\%), followed by emollient (21.2\%). Out of total of 905 drugs 229 (25.3\%) were Among topical drugs, Betamethasone cream/ointment was the most common prescribed drug. The most commonly prescribed drug was ketoconazole shampoo (50.9\%) from the anti-fungal and doxycycline (37.7\%) from anti-biotic (Table 6).

Out 385 prescription of drugs prescribed $67.5 \%$ frequency of application, $56.1 \%$ duration of treatment and $54.0 \%$ site of applications are specified. Consideration of various parameters while prescribing in the prescription of drugs (Table 7).

\section{Discussion}

The results of this study provide valuable information on the prescribing patterns of doctors for dermatological patients at $\mathrm{BMH}$.

Many of the patients seen were in male between the age group of 21 - 30 years (25.5\%) and in female $21-30$ years $(32.6 \%)$. This results were compare with in 
Table 2. Distribution of prescription item in Generic/Brand name in dermatology unit of BMH, 2016.

\begin{tabular}{cccc}
\hline Prescription item & Generic & Brand & Total \\
\hline Single drug & $572(81.7 \%)$ & $128(18.3 \%)$ & 700 \\
Combination drug & $205(84.4 \%)$ & $38(15.6 \%)$ & 243 \\
\hline
\end{tabular}

Table 3. Route of administration number of drugs prescribed in dermatology unit of BMH, 2016.

\begin{tabular}{ccc}
\hline Route of administration & Number & Percentage (\%) \\
\hline Oral & 212 & $19.2 \%$ \\
Topical & 731 & $66.2 \%$ \\
Parenteral & 1 & $0.1 \%$ \\
Combination (systemic \& local) & 160 & $14.5 \%$ \\
Total & 1104 & $100 \%$ \\
\hline
\end{tabular}

Table 4. No. of drugs per prescription in dermatology unit at BMH, Dessie, 2016.

\begin{tabular}{cc}
\hline No. of drugs per prescription & No. of prescription, $\mathrm{n}(\%)$ \\
\hline 1 & $57(14.8 \%)$ \\
3 & $180(46.8 \%)$ \\
4 & $87(22.6 \%)$ \\
5 & $42(10.9 \%)$ \\
6 & $16(4.2 \%)$ \\
7 & $1(0.3 \%)$ \\
No. of drugs per prescription & $2(0.5 \%)$ \\
\hline
\end{tabular}

Table 5. Disease patterns in dermatology unit of BMH, 2016.

\begin{tabular}{cccc}
\hline Disease & Male, $\mathrm{n}(\%)(467)$ & Female, $\mathrm{n}(\%)(456)$ & Total \\
\hline Atopic dermatitis & $123(26.3 \%)$ & $95(20.8 \%)$ & $218(47.1 \%)$ \\
Acne vulgaris & $34(7.3 \%)$ & $59(12.9 \%)$ & $93(20.2 \%)$ \\
Nummular eczema & $44(9.4 \%)$ & $48(10.5 \%)$ & $92(19.9 \%)$ \\
Sebohoric dermatitis & $31(6.6 \%)$ & $45(9.9 \%)$ & $76(16.5 \%)$ \\
Scabies & $57(12.2 \%)$ & $17(3.7 \%)$ & $74(15.9 \%)$ \\
Tinea captious & $24(5.1 \%)$ & $29(6.4 \%)$ & $53(11.5 \%)$ \\
Allergic contact dermatitis & $14(3 \%)$ & $33(7.2 \%)$ & $47(10.2 \%)$ \\
Pityriasis alba & $23(4.9 \%)$ & $17(3.7 \%)$ & $40(8.6 \%)$ \\
Supper infected eczema & $22(4.7 \%)$ & $16(3.5 \%)$ & $38(8.2 \%)$ \\
Melisma & $11(2.4 \%)$ & $26(5.7 \%)$ & $37(8.1 \%)$ \\
Psoriasis & $13(2.8 \%)$ & $19(4.2 \%)$ & $32(7 \%)$ \\
Tinea pedis & $27(5.8 \%)$ & $4(0.9 \%)$ & $31(6.7 \%)$ \\
Vitiligo & $13(2.8 \%)$ & $16(3.5 \%)$ & $29(6.3 \%)$ \\
Cellulites & $18(3.9 \%)$ & $10(2.2 \%)$ & $28(6.1 \%)$ \\
Impetigo & $4(0.9 \%)$ & $17(3.7 \%)$ & $21(4.6 \%)$ \\
Onchomycosis & $9(1.9 \%)$ & $5(1.1 \%)$ & $14(3 \%)$ \\
\hline
\end{tabular}


Table 6. Category of drugs prescribed at the dermatology unit of BMH, Dessie, 2016.

\begin{tabular}{ccc}
\hline Drug category & No, of drug & Percentage \\
\hline Topical steroid & 229 & $25.3 \%$ \\
Emollient $^{*}$ & 192 & $21.2 \%$ \\
Anti-biotic & 149 & $16.5 \%$ \\
Antifungal & 113 & $12.5 \%$ \\
Systemic steroid & 29 & $3.2 \%$ \\
Antihistamine & 24 & $2.7 \%$ \\
Vitamins & 3 & $0.3 \%$ \\
Anti-viral & 2 & $0.2 \%$ \\
Others* & 164 & $18.1 \%$ \\
Total & 905 & $100 \%$ \\
\hline
\end{tabular}

${ }^{*}$ emollient like: liquid paraffin, white petrolatum ointment, Neiva cream; ${ }^{*}$ others such as: salicylic acid powder, urea powder, metronidazole powder.

Table 7. Various parameters in the prescriptions of drug in dermatology unit of BMH, 2016.

\begin{tabular}{ccc}
\hline Parameters & No, of prescriptions on which specified & Percentage (\%) \\
\hline Frequency of application & 260 & $67.5 \%$ \\
Duration of treatment & 216 & $56.1 \%$ \\
Site of application & 208 & $54.0 \%$ \\
\hline
\end{tabular}

the past study done in Ayder referral hospital, which were maximum numbers of patients in male between the age group of $11-20$ years $(32.14 \%)$ and in female 11 - 20 years $(31.63 \%)$ [6].

Number of drugs per prescription varied from 1 to 7 with average of 2.46 . Most of the prescriptions consists of minimum of 2 drugs (180 prescriptions, $46.8 \%$ ). And the others with $14.8 \%, 22.6 \%$ contain a single drug and 3 drugs respectively. This study showed the incidence of poly pharmacy. Poly pharmacy is the use of an excessive number of inappropriate medicines. Average number of drugs per prescription reported in this study was very low as compared to studies by Sarkar C et al., and Narwane SP et al., where average number of drugs prescribed were lesser than 3 drugs per prescription i.e., 2.7 and 2.64/prescripttion respectively [7] [8].

This study showed that atopic dermatitis (26.3\%) and (20.8\%) was the most common dermatological condition in the dermatology unit in both male and female respectively, Followed by scabies (12.2\%) in male and acne vulgaris $(12.9 \%)$ in female. different findings were also reported from study carried out in India the study showed fungal disease was the most common [9]. From the total of 1104 drugs prescribed, Topical corticosteroids were accounts for731 (66.2\%) followed by emollient (21.2\%). This finding was similar with topical corticosteroids in Ayder referral hospital study and different from a study done 
by Bijoy KP et al. and M Ashok Kumar et al in India where Anti-fungal were the most commonly prescribed [9] [10].

It is important that drugs should be prescribed in their generic names to avoid confusion and minimize the costs. But the result of the current study showed $18.3 \%$ of a single drug and $15.6 \%$ of drugs in fixed dose combinations were prescribed with brand name. This finding was different with the study done by Ayder referral hospital study showed that $94.57 \%$ of a single drug and $100 \%$ of drugs in fixed dose combination were prescribed with brand name [6].

The higher percentage of nonproprietary prescriptions in outpatients invokes the problem of the vast expenditure on such drugs and their availability in the hospital pharmacy. Many patients attending a hospital as out-patients belong to the lower socio-economic status and would have to buy nonproprietary drugs from a low price. But he/she would have to buy proprietary drugs from high price and other relevant pharmacy outlets. Lack of trust in the quality of generic drugs could be the reason why brand names were highly prescribed.

Among the total number of drugs prescribed, most of them were prescribed by topical (66.2\%) followed by oral routes (19.2\%) and the second most route of administration were a combination of systemic and local route of administration (14.5\%). The reason for a high percentage of topical drugs being pre scribed is that topical route has minimum side effects hence is the preferred route of administration in dermatology. The use of fixed-Dose combinations may help to bring down the cost and improve compliance. According to salwaomer et al. study combination route of administration (systemic and local) was highly prescribed with a prevalence of $67.2 \%$ followed by oral route which was prescribed for $25.3 \%$ of cases [11].

Frequency of application was specified only in $67.5 \%$ of prescriptions and duration of treatment and site of application was also steted in $56.1 \%$ and $54.0 \%$ of prescriptions respectively. This finding was similar with the study done by Ayder referral hospital, M Ashok Kumar et al and W.M Sweileh [6] [9] [12].

\section{Conclusion}

The current study reveals that average number of drugs per prescription was high (2.46) and topical corticosteroids were commonly prescribed drugs (25.3\%) in the dermatology unit. This finding also showed that atopic dermatitis was the most prevalent disease condition. Therefore, trainings and workshops should be organized from time to time to enlighten the prescribers on the current trend in dermatological treatments. Similar studies should also be carried out in other health care facilities, in order to help the stakeholders to identify possible problems with a view of finding lasting solutions, which will enhance rational drug use and routine auditing of prescriptions will be helpful for the improvement of proper use of drugs which in turn can provide good quality of health care.

\section{Limitations}

The study tolls not fully supported by WHO prescribing indicators, which were 
not assess percentage of antibiotic use and prescribed with in facility/national drug list. The prescribing indicators measure aspects of outpatient treatments, other techniques are needed where the drug use pattern is more complex especially dermatology clinic.

\section{References}

[1] World Health Organization (2006) Epidemiology and Management of Common Skin. WHO, Geneva.

[2] Benskin, L.L.L. (2013) Diseases in Children in Developing Countries. 1-42.

[3] Chester, B.G. (2002) Poly Pharmacy in Elderly Patients with Diabetes. Diabetes Spectrum, 15, 240-248. https://doi.org/10.2337/diaspect.15.4.240

[4] Michael, J.C., John, T., Catherine, H., Julie, C., Victoria, B., Rachid, T.A., et al. (2003) An Audit of Adverse Drug Reactions to Aqueous Cream in Children with Atopic Eczema. International Journal of Pharmaceutics, 271, 747-748.

[5] Siti, F., Maksum, R. and Retnosari, A. (2017) Polypharmacy in Elderly Patients and Their Problems. Asian Journal of Pharmaceutical and Clinical Research, 10, 44-49.

[6] Hafta B.M., Martha, K. and Minyahil, A.W. (2014) Prescribing Pattern in Dermatology Unit of Ayder Referral Hospital. World Journal Pharmaceutical Research, 4, 17.

[7] Manjusha, S., Kaveri, D.L., Soumya, P. and Pawar, A.P. (2014) Prevalance of Various Skin Disorders and Prescribing Pattern of Antihistamines in Tertiary Care Hospital, Pune. International Journal of Pharma Sciences and Research, 5, No. 3.

[8] Audit Commission (1994) A Prescription towards More Rational Prescribing in General Practice. HMSO, London.

[9] Ashok, M., Kumar, P.P., Noushad, K., Shailaja, Jjayasutha, C. and Rmasamy (2011) A study on Drug Prescribing Pattern and Use of Cortico Steroids in Dermatological Conditions at a Tertiary Care Teaching Hospital. International Journal of Pharmaceutical Sciences Review and Research, 9, 69-71.

[10] Bijoy, K.P. (2012) Drug Prescribing and Economic Analysis for Skin Diseases in Dermatology OPD of an India Tertiary Care Teaching Hospital: A Periodic Audit. Indian Journal of Pharmacy Practice, 5, 28-33.

[11] Salwa, O.B., Gamila, H. and Mohamed, A. (2016) Prescription Patterns of Antibiotics in Five Dermatologic Outpatient Clinics; A Cross Sectional Study from Yemen. Journal of Pharmacy Practice and Community Medicine, 2, 65-69. https://doi.org/10.5530/jppcm.2016.3.2

[12] Sweileh, W.M. (2014) Audit of Prescribing Practices of Topical Corticosteroids in Outpatient Dermatology Clinics in North Palestine. Eastern Mediterranean Health Journal, 12, 161-163. 